\title{
Culturas del malgasto público: ineficiencia estatal y narrativas de política pública
}

\section{Erick Behar-Villegas 1}

${ }^{1}$ Berlin International University of Applied Sciences, Berlin - Alemania

\begin{abstract}
El artículo introduce el concepto de las culturas del malgasto público en el marco del estudio de la ineficiencia estatal. Estas culturas engloban comportamientos aprendidos en el trabajo inercial de la administración pública. Utilizando un estudio de caso sobre el malgasto en camionetas blindadas para el Estado, en Colombia, se ilustran las culturas del malgasto. El rol de las narrativas de políticas públicas es crucial para comprender dichas culturas, al sustituir y relegar discusiones técnicas sobre la ineficiencia a un segundo plano, favoreciendo tradiciones institucionales que incluyen el incrementalismo. El artículo contribuye a la literatura interdisciplinaria sobre la ineficiencia estatal, incorporando el rol del storytelling, las narrativas, y los cálculos de costo-efectividad. Resalta, desde el estudio de caso, la necesidad de que la política pública comprenda las complejidades que rodean a las prácticas del gasto público para así mejorarlo.
\end{abstract}

Palabras clave: eficiencia estatal; narrativas de políticas públicas; vehículos blindados; malgasto público; cultura.

\section{Culturas do mal gasto público: ineficiência do Estado e narrativas de políticas públicas}

$\mathrm{O}$ artigo introduz o conceito de culturas do mal gasto público no âmbito do estudo da ineficiência do Estado. Essas culturas abrangem comportamentos aprendidos no trabalho inercial da administração pública. As culturas do mal gasto são ilustradas através de um estudo de caso sobre o mal gasto em compras públicas de carros blindados para o Estado na Colômbia. O papel das narrativas de políticas públicas é crucial para a compreensão dessas culturas, substituindo e relegando as discussões técnicas sobre ineficiência para o segundo plano, favorecendo assim tradições institucionais que incluem o incrementalismo. $\mathrm{O}$ artigo contribui para a literatura interdisciplinar sobre a ineficiência do Estado, incorporando o papel do storytelling, das narrativas e dos cálculos de custo-efetividade. Destaca, a partir do estudo de caso, a necessidade de políticas públicas para entender melhor as complexidades que envolvem as práticas de gasto público, a fim de melhorá-o.

Palavras-chave: eficiência do Estado; narrativas de políticas públicas; veículos blindados; gastos públicos; cultura.

\section{Wasteful spending cultures: State inefficiency and policy narratives}

The article introduces the concept of "wasteful public spending cultures" in the framework of state efficiency studies. The concept refers to learned behavioral patterns in the inertial work of government agencies. A case study from Colombia is presented. The study reports practices that lead to the wasteful procurement of armored cars to be used by the government. The role of policy narratives is crucial to understanding these "cultures," as they contribute to replacing and downgrading technical discussions about inefficiency, favoring institutional traditions that include incrementalism. The work contributes to the interdisciplinary body of literature on government inefficiency, as it incorporates the role of storytelling, narratives, and cost-effectiveness calculations. The case study highlights the need to understand the complexity around spending practices in order to improve them.

Keywords: State efficiency; policy narratives; armored government cars; wasteful spending; culture. 


\section{INTRODUCCIÓN}

Durante la pandemia del COVID-19 en 2020, la Fiscalía General de Colombia (2020) anunció investigaciones masivas por malgasto público. Poco tiempo después, se reportó que la misma Fiscalía y otros organismos de control también habrían incurrido en prácticas parecidas de malgasto (González Gaitán, 2020). La complejidad de la realidad y del concepto del malgasto público puede ser estudiada explorando casos puntuales, que sugieren la existencia de culturas, narrativas y tendencias en la administración pública de países en desarrollo. Su comprensión ofrece un punto de partida para tematizar problemáticas latentes y posibles soluciones.

En las Memorabilia de Jenofonte (1997/s.d), Sócrates increpa a Glaucón, que aspira llegar al poder en Atenas. "Por lo menos cuéntanos sobre el gasto de la ciudad. Pues es claro que quieres remover aquellos gastos superfluos. Pero, por Zeus, respondió [Glaucón], tampoco he tenido aún ocio para aquello". La preocupación de Sócrates recuerda la importancia de la eficiencia en el gasto público. Esta implica hacer "más con menos", ya sea desde la visión de minimización de inputs o maximización de outputs (Rueda López, 2011), sin olvidar que "más" implica también un gasto de mejor calidad (Izquierdo, Pessino \& Vuletin, 2018).

El malgasto público en América Latina es un problema estructural, que se relaciona con preocupaciones como la sostenibilidad fiscal, la calidad de vida y la desigualdad (Izquierdo et al., 2018). El malgasto también ha sido asociado con la corrupción (Dal Bó \& Rossi, 2007; Rocha Menocal \& Taxell, 2015; Rose-Ackerman, 1997). Las ineficiencias del gasto público en América Latina pueden costar hasta el 4.4\% del PIB regional (Banco Interamericano de Desarrollo, 2018). Sin embargo, ¿qué sucede si parte del malgasto no se registra en dichas estadísticas, dado que se derivaría de costumbres administrativas difícilmente identificables?

Este trabajo se propone varios objetivos. Primero, plantea un marco conceptual para aproximarse al problema del malgasto público, desde aquello que llamaré las "culturas del malgasto", que se encuentran ancladas y protegidas por narrativas (e.g. la seguridad). Segundo, busca ilustrar y caracterizar la complejidad en la identificación de dichas costumbres del malgasto, utilizando un estudio de caso sobre compras públicas de vehículos blindados Tercero, se busca contribuir, por un lado, al creciente cuerpo de literatura sobre las narrativas y las políticas públicas (cf. Sección 3) y, por otro, a enriquecer la literatura interdisciplinaria sobre la eficiencia estatal en Latinoamérica, integrando el rol de narrativas y comportamientos aprendidos, a través del estudio de caso. Si bien este estudio se enfoca en Colombia, la misma realidad puede estudiarse en demás países del continente, que guardan semblanzas en su desarrollo institucional, en su dimensión cultural y en preocupaciones generales como la corrupción, la intransparencia y la baja productividad. Por ejemplo, y, en relación al estudio de caso que aquí se presenta, hace parte de la normalidad que se adquieran vehículos blindados de alta gama en países como México y Brasil (cf. Cantera, 2018; Montoia, 2019), sin que se presenten estudios concretos y estructurales sobre mejoras cualitativas que van más allá de anuncios de reducción absoluta de rubros (e.g. el caso de México en 2018, cuando la Presidencia anunció una política de austeridad).

El artículo está estructurado de la siguiente manera. La sección 2 aporta unas consideraciones metodológicas sobre el uso de estudios de caso. Luego, la sección 3 introduce el concepto de "culturas del malgasto" y su practicidad en el análisis de lo público, ofreciendo un marco conceptual que vincula el rol de las narrativas. En la sección 4 se presenta el estudio de caso sobre el uso de camionetas blindadas de alta gama en el Estado, desde un análisis de costos y de poder adquisitivo relativo. 


\section{CONSIDERACIONES METODOLÓGICAS}

Los estudios de caso ofrecen una ventaja metodológica ligada a la narración de un problema, dando acceso a complejidades que difícilmente se pueden entender desde la agregación de datos que se usan para construir indicadores. Estos últimos también suponen un problema, pues calcularlos implica que abarcan definiciones claras de lo que es y no es gasto de calidad. Sin embargo, la parte menos visible de la realidad se escapa al no poderse cuantificar y sistematizar como gasto ineficiente. Esto implica que hay una naturaleza casuística en el malgasto público, en donde las reglas generales (e.g. austeridad medida en cambios porcentuales) no logran captar la realidad.

Para ilustrar esta problemática, se puede tomar el caso analizado en este estudio. Si no se considera un problema la compra de un vehículo de alta gama para el Estado, ¿cómo se ha de cuantificar y agregar su diferencial contra vehículos inferiores, a las cifras de ineficiencia? Esta dificultad aumenta en presencia de narrativas que frenan los cuestionamientos de rubros específicos. En el nombre de la seguridad, se compran los vehículos, pero increpar las especificidades de las compras se hace difícil ante narrativas robustas. Los estudios de caso capturan la complejidad de algo en el marco de “circunstancias importantes" (Stake, 1995, p. xi), y pueden revelar distintas prácticas y culturas de la gestión pública, enriqueciendo las posibilidades de generar recomendaciones y fortalecer el trabajo cualitativo y cuantitativo sobre el gasto. El método no es ajeno al estudio de lo público (cf. Hauptmeier, Heinpertz \& Schuknecht, 2006; PwC, 2012; Shenggen, 2007), aunque en el caso latinoamericano, más allá de la prensa, su uso es relativamente escaso (cf. Doimeadiós Reyes \& Rodríguez Llorian, 2015).

\section{CULTURAS DEL MALGASTO PÚBLICO: CONTEXTO Y NARRATIVAS}

Tematizar el complejo concepto de cultura (cf. Moosmüller, 2000) usualmente implica traer a colación acervos de creencias, significados, rituales, comportamientos, tradiciones y demás elementos que se pueden encontrar a nivel nacional (Licht, Goldschmidt \& Schwartz, 2005) u organizacional (Mastroianni, 2006; Ouchi, 1980; Schein, 1990), entre otros niveles. El concepto de cultura implica la interacción y el aprendizaje entre seres humanos (Hannerz, 2010). En este trabajo no se entiende la cultura como el concepto alemán de Kultur, que se acerca más a la idea de civilización, sino a la idea de contexto y estructuras de significados (Geertz, 1973).

El concepto de cultura ha sido previamente vinculado al malgasto. Payne (1991) se refiere a una cultura del gasto (Culture of Spending) en Estados Unidos desde la óptica del Congreso, que busca gastar más allá de sus medios, pensando que el gobierno será eficaz solucionando los problemas. Mostrando cómo desde el Congreso se ignora el mandato constitucional del presupuesto equilibrado, argumenta que los legalismos no necesariamente solucionan el problema del gasto. Mientras que su perspectiva parte de una idea de excesos sobre capacidades, en este artículo se busca entender las complejidades de cómo y por qué se gasta con menor eficiencia de la que se podría alcanzar.

En este trabajo se propone tematizar las culturas relacionadas con el gasto público, sobre todo si se piensa en la diversidad de condiciones que rodean a cada funcionario(a). Estas culturas responden, entre otros, a narrativas de política pública (policy narratives) que guían la formulación de planes de desarrollo, iniciativas legislativas y demás instrumentos disponibles en el Estado, a través de "sistemas de historias" (Cormann, 2013). En estos sistemas pueden existir narrativas técnicas, que fungen como secuencias de acciones o eventos, atados a un manual, para utilizar el concepto de Schank y Abelson 
(1977). Los tecnicismos que se utilizan en un proceso administrativo pueden responder a narrativas, que paradójicamente son difíciles de encontrar a simple vista.

El uso de las historias (storytelling) se devuelve a la antigüedad e ilustra cómo las realidades se moldean en relatos ${ }^{1}$. Remontarse a la tradición oral de Heródoto en la Grecia Antigua, o a los trabajos escritos durante la dinastía Qin en la China imperial del siglo III A.C, ilustra el concepto de homo-narrans (Jones, McBeth \& Shanahan, 2014). El estudio de las policy narratives, sin embargo, es reciente y algo distinto (cf. Jones \& McBeth, 2010; Lowndes, 2016; Polletta, 2008; Stone, 1988). Hablar de "culturas del malgasto público" requiere la comprensión de la interrelación que hay entre comportamientos aprendidos y narrativas. Los hacedores de políticas públicas no realizan necesariamente una evaluación racional de costos y beneficios para tomar decisiones, usando al final reglas cognitivas o heurísticas, que reflejan narrativas, o lo que Mishler (1995) llama "historias compartidas culturalmente", que a su vez se derivan de relaciones de poder (Lowndes, 2016).

El contexto o escenario (setting) en el que se desarrolla la administración pública, puede contribuir a la generación de comportamientos que se convierten en tradiciones, de las cuales hay o no consciencia en el actuar individual. Vale la pena mencionar el reciente desarrollo que ha tenido el Narrative Policy Framework, que se orienta hacia la comprensión de escenarios, y no solo de elementos aislados de narrativas de política, como los personajes o las creencias (French, Shanahan, Raile \& McEvoy, 2017). Comprender el escenario, que a su vez influencia el actuar de un funcionario público, puede ayudar a discernir la manera en que se van tejiendo patrones de comportamientos aprendidos (Hiebert, 1997). Estos tejen culturas administrativas que devienen en normas y complementan los rígidos mecanismos burocráticos bajo los cuales opera la administración pública (Ouchi, 1980).

Partir de los comportamientos pasados y las tradiciones institucionales puede significar una inercia administrativa que va en contra de la innovación estatal. En el estudio de narrativas de política pública, se ha encontrado cómo la comprensión de una historia nueva está anclada a historias pasadas (Polletta, 2008, p. 28). Esto puede dificultar el establecimiento de nuevas perspectivas innovadoras que contribuyan a un "gasto inteligente" (Izquierdo et al., 2018, p. 54). Existe, por ejemplo, la práctica del incrementalismo, cuando "las decisiones presupuestarias están ancladas en el pasado, y normalmente varían solo en pequeños incrementos de un año al siguiente" (Izquierdo et al., 2018, p. 320). Esta costumbre presupuestaria se vuelve un refugio y una tradición, protegida por sistemas legales, presiones políticas, narrativas técnicas y falta de experticia, que no permiten proyectos de alto riesgo y largo alcance.

\subsection{Conceptualizando las Culturas del Malgasto Público}

El malgasto público (wasteful spending) ha sido tematizado frecuentemente en la prensa (El Mostrador, 2008; Revista Semana, 2020a; Rodríguez, 2019), en la literatura científica (Bandiera, Prat \& Valleti, 2009; Holdsworth, 2019; Liebman \& Mahoney, 2018) e inclusive en iniciativas ciudadanas (e.g. Citizens Against Government Waste, 2015). Cualquier aproximación conceptual que aborde los comportamientos aprendidos, relacionados con el malgasto, parte de una realidad relativa, que se asocia al escenario, las capacidades de los funcionarios o el momento (e.g. una pandemia, tiempos

\footnotetext{
${ }^{1}$ Para ver la diferencia entre historia y narrativa, cf. Cormann (2013).
} 
electorales, etc.). De ahí que se proponga conceptualizar el malgasto desde lo plural, pensando que no hay una, sino varias culturas superpuestas, en distintos niveles de gobierno, que engloban los patrones de comportamientos aprendidos que rodean el malgasto.

El malgasto está ligado a la ineficiencia estatal, que puede ser técnica, i.e. no hacer las cosas bien con los recursos disponibles, o asignativa, i.e. hacer bien las cosas equivocadas (Izquierdo et al., 2018). El reto que suponen los ciclos políticos, la inercia cortoplacista, la falta de experticia, así como prácticas ancladas que no necesariamente se cuestionan, implica que hay un largo camino por recorrer en el estudio de la ineficiencia estatal. Mientras que el malgasto activo puede asociarse, entre otras, a la corrupción, al mejorar la situación del servidor público, el malgasto pasivo implica no alcanzar los objetivos que se trazaron en una política pública, sin necesariamente lucrar al servidor (cf. Bandiera et al., 2009).

Al exponer lo necesario para lograr una buena relación calidad-precio en el gasto público, McKevitt (2015 como citado en Izquierdo et al., 2018, p. 315), menciona tres principios: la economía (cuando se adquieren bienes y servicios en cantidades y calidades adecuadas), la eficiencia (pagar el costo mínimo por el mismo servicio), la efectividad (lograr los resultados deseados). Las culturas del malgasto público se materializan en la violación de uno o varios de estos principios, partiendo de comportamientos anclados en tradiciones (e.g. comportamientos corruptos de privados y públicos, incrementalismo presupuestal, baja innovación en procesos y proyectos, etc.). En otras palabras, las culturas del malgasto público son posibles escenarios aprendidos, prácticas, tradiciones y comportamientos que disminuyen la calidad-precio en los resultados de la gestión pública.

El malgasto funge como una práctica inconveniente para la consecución de objetivos legitimados socialmente, e.g. eficiencia para mejorar la calidad de vida. Conceptualmente, el malgasto y las culturas que lo permean se pueden entender como un costo de oportunidad social (cf. Behar-Villegas \& Amado, 2021), ampliado o influenciado por narrativas y contextos que se reflejan en historias y tradiciones aprendidas. Si se incurre en una práctica cuestionable desde un objetivo mayor (e.g. la transparencia), es posible que esa práctica esté ligada a gastos mayores que podrían haberse destinado a otros rubros en el Estado (e.g. un hospital). La problemática aumenta cuando el malgasto no se percibe como tal, dado que es justificado automáticamente por una narrativa que también proviene de un objetivo mayor (e.g. la paz). Sin embargo, en nombre de una narrativa se puede incurrir en costos de oportunidad.

Un riesgo que tiene la administración pública es la politización de la identificación de estos costos de oportunidad, pues ella implicará la probable inclusión de otra narrativa, que al final, opera como un velo que frena un análisis equilibrado y transparente del problema. En términos cuantitativos, una vez se identifica el malgasto como práctica, se pueden introducir variables medibles para entender la magnitud del costo de oportunidad. La dificultad en la concreción metodológica de este concepto aparece en su naturaleza casuística, pues de cada escenario estudiado, se requiere comprender en dónde puede estar el costo de oportunidad (e.g. gasto en rubro x, diferencial de gasto, gastos de corto plazo vs. largo plazo, etc.).

Como ejemplos de estas prácticas se pueden mencionar el aumento drástico del gasto a finales de año en proyectos menos relevantes (Liebmann \& Mahoney, 2018), el pago de publicidad personal indirecta para los mandatarios vía marcas de gobierno (Behar-Villegas, 2018) o el sacrificio de proyectos de largo plazo que implican incertidumbre, en pro de proyectos de corto plazo, capitalizables política, pero no socioeconómicamente. 


\section{ESTUDIO DE CASO: ¿SON NECESARIAS LAS CAMIONETAS BLINDADAS DE ALTA GAMA EN EL ESTADO COLOMBIANO?}

\subsection{Introducción}

La disponibilidad de información sobre compras públicas en Colombia ha conocido ligeras mejoras. La existencia de las compras públicas electrónicas (e-procurement), a través del sistema SECOP (Servicio Electrónico de Contratación Pública), brinda acceso a datos de contratación en distintos niveles de gobierno, y permite generar alertas y pronósticos (cf. Gallego, Rivero \& Martínez, 2020). Con la creación de la agencia de agregación de demanda y compras públicas, Colombia Compra Eficiente (CCE) en 2011 y la introducción de Acuerdos Marco de Precios (AMP), se ha buscado crear más transparencia y eficiencia como contrapeso a la asimetría de información existente en la contratación pública.

En este caso se aborda el estudio del gasto público desde el principio corporativo de presupuesto base cero (zero-based budgeting), que, al iniciar cada año, pregunta si hay justificación adecuada para cada uno de los gastos a asumir. La dificultad que supone juzgar como gasto o malgasto cualquier actuación o rubro se refleja en la difusa definición de lo 'adecuado'. Sin embargo, en este artículo se parte de lo expuesto arriba sobre los tres principios de McKevitt (2015) para una buena relación calidad-precio en las compras públicas (economía, eficiencia y efectividad).

\subsection{Camionetas blindadas y la tradición aprendida}

Es común ver caravanas de carros blindados de alta gama en las calles colombianas, algunos al servicio del Estado, y otros de uso privado. Este caso se basa en algunos de los resultados del proyecto de investigación interdisciplinaria sobre efectividad y eficiencia en el gasto público en camionetas blindadas en Colombia (Behar-Villegas \& Amado, 2021). Dicho proyecto parte, entre otras, de esta pregunta: ¿Es necesario tener camionetas blindadas de alta gama en el Estado?

Hablar de lo necesario es relativo, pues supone, en el estudio de costo beneficio del Estado, que se alcance un objetivo particular, por ejemplo, proteger a una persona. Si se analiza la diferencia entre la adquisición de un vehículo de alta gama contra uno de gama media, a la luz de la misión de garantizar la seguridad de la persona, se puede tematizar el ahorro en las compras públicas. Sin embargo, el obstáculo que tienen las prácticas de eficiencia se encuentra en la narrativa subyacente de la seguridad, que eclipsa cualquier cuestionamiento de las compras públicas a un nivel detallado. El uso de la narrativa de la seguridad puede sustituir y volver irrelevante la discusión técnica de detalles.

En Latinoamérica y el Caribe, $30 \%$ del gasto público corresponde a las compras públicas, que fungen como epicentro potencial de corrupción y/o ineficiencias, perdiendo hasta una tercera parte del dinero público vía malgasto (Izquierdo et al., 2018). El caso de los vehículos de alta gama se suma a dicho valor, dado que los tecnicismos, que llevan a la compra o alquiler de estos elementos, nublan el análisis de costo beneficio del gasto en los estudios previos a la contratación. De ahí que difícilmente se pueda hablar de sobrecostos, si la categoría del carro no se cuestiona. ¿Qué sucede, sin embargo, si se pueden alcanzar los mismos objetivos con carros menos costosos? 
Si se encuentran ineficiencias en este tema particular, ¿cómo se pueden documentar y sistematizar en los cálculos del malgasto?

En este aparte se pregunta si los vehículos que se compran responden al óptimo de efectividad de costos alcanzable, implicando que la misión del vehículo se debe cumplir con calidad, mientras que los costos se minimizan. Para ejemplificar la problemática, se puede percibir la complejidad de la situación en la siguiente pregunta, ¿es adecuado que algunos funcionarios públicos se movilicen en camionetas de alta gama que superan los 70.000 USD?

\subsubsection{Introducción al caso: cifras del mercado automotor en Colombia}

Para el 2019, un estudio del reconocido portal Tucarro.com, arrojó un precio promedio de compra de vehículo en Colombia de 11.000 USD. En el mismo estudio se clasifican los vehículos en tres categorías o gamas: baja, media y alta. En la Figura 1 se pueden ver algunos ejemplos de modelos por categoría, con sus respectivos precios, tanto en usados como nuevos, según fuentes como la Revista Motor, que registra mensualmente los precios de usados y nuevos. Si se toman vehículos típicos utilizados por altos cargos del Estado, es evidente que se trata de vehículos reconocidos como de gama alta. Paradójicamente, al preguntarle formalmente a varias entidades del Estado, vía derechos de petición, si contaban con vehículos de alta gama, se recibieron negaciones contundentes.

\section{FIGURA 1 RANGOS DE PRECIOS DE VEHÍCULOS EN COLOMBIA}

\begin{tabular}{|c|c|c|c|c|}
\hline $\begin{array}{c}\text { Categoría Portal } \\
\text { Tucarro.com }\end{array}$ & Ejemplo Vehículo & $\begin{array}{c}\text { Vehículos más buscados } \\
\text { en la categoría }\end{array}$ & $\begin{array}{l}\text { Rango de precios Nuevos } \\
\text { (2019) precios en USD }\end{array}$ & $\begin{array}{c}\text { Rango de precios Usados } \\
\text { (2019) precios en USD }\end{array}$ \\
\hline Gama baja & $\begin{array}{l}\text { Ford Fiesta, KIA Picanto, } \\
\text { Renault Logan, Peugeot } \\
208\end{array}$ & $\begin{array}{l}\text { Chevrolet Spark, Spark } \\
\text { GT, R. Twingo }\end{array}$ & $10.350-18.500$ & $3.350-5.485$ \\
\hline Gama media & $\begin{array}{l}\text { Chevrolet Tracker, Nissan } \\
\text { X-Trail, Mazda 3, Ford } \\
\text { Escape, Suzuki Vitara }\end{array}$ & $\begin{array}{l}\text { Mazda 3, KIA New } \\
\text { Sportage,. } \\
\text { Chevrolet LUV }\end{array}$ & $20.500-36.500$ & $12.200-16,765$ \\
\hline Gama alta & $\begin{array}{l}\text { Toyota Fortuner, Audi } \\
\text { A5, Mercedes Clase E,C, } \\
\text { Porsche Cayenne. Audi } \\
\text { Q7, BMW X3 }\end{array}$ & $\begin{array}{l}\text { Toyota Prado, Hilux, } \\
\text { Fortuner }\end{array}$ & $\begin{array}{l}53.400-62.500 \\
\& \text { superior }\end{array}$ & $\begin{array}{l}25,900-40,550 \\
\& \text { superior }\end{array}$ \\
\hline
\end{tabular}

Fuente: Elaborada por el autor basada en Tucarro.com, Revista Motor, Autocosmos.com, Promedio TRM: Banco de la República, Cálculos propios. 


\subsubsection{Poder adquisitivo e ineficiencia en el gasto}

Si se compara el poder adquisitivo de Colombia con Japón y Estados Unidos, países de origen de las empresas que venden algunos de los vehículos que tradicionalmente se blindan, aparece una brecha típica entre países de ingreso medio y alto. Al tomar los precios equivalentes de los mismos vehículos en su mercado de origen, para contrastarlos con el poder adquisitivo correspondiente de los tres países, se puede responder a los siguientes interrogantes: ¿cuántas veces se debe multiplicar la capacidad de pago de un hogar para adquirir un vehículo determinado en cada país? La Figura 2 presenta el caso de la Chevrolet Tahoe y la Toyota Land Cruiser Imperial en Colombia, Estados Unidos y Japón.

\section{FIGURA 2 COMPARATIVO DE PODER ADQUISITIVO EN HOGARES EE.UU/JAPÓN/COLOMBIA}

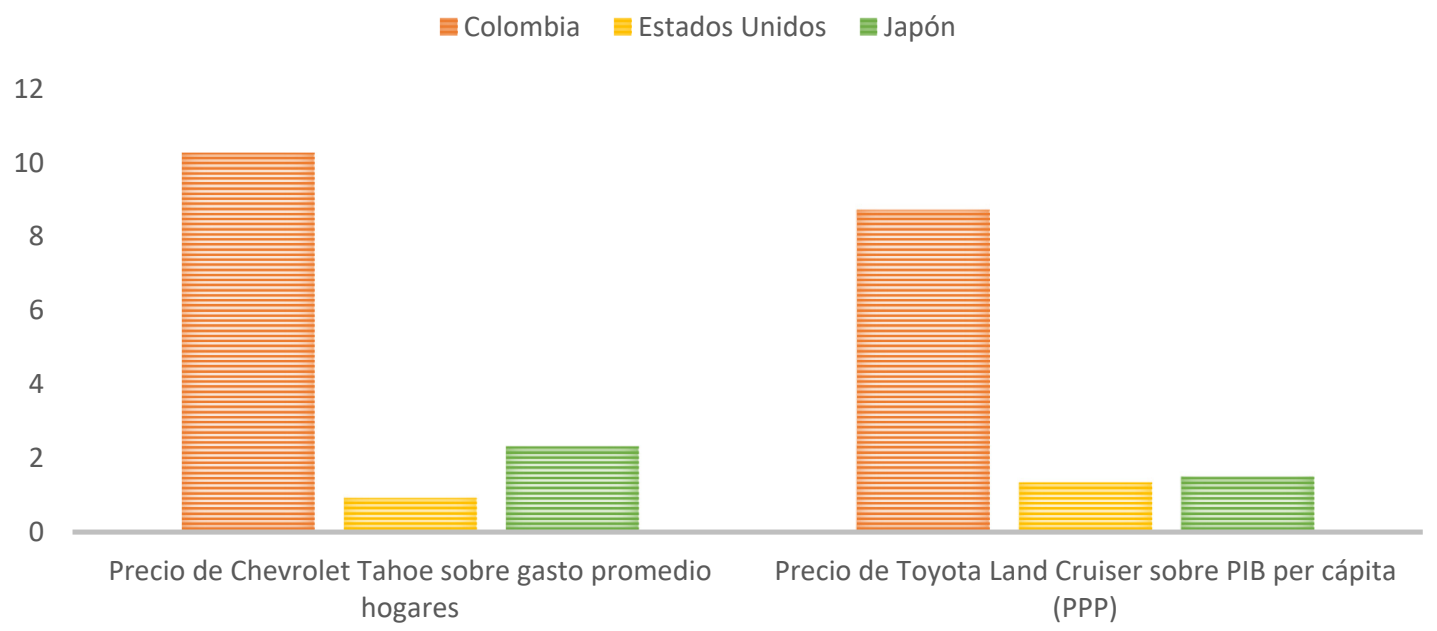

Nota: Las barras de la izquierda muestran el cociente entre el precio nominal de una camioneta Tahoe en cada país y el gasto promedio anual de un hogar del país respectivo. Las barras de la derecha muestran el cociente entre el precio de otra camioneta sobre el PIB per Cápita en dólares PPA-PPP.

Fuente: Elaborada por el autor a partir de datos de DANE, Kelly Blue Book, Kakaku Japan, Tucarro.com, y varios portales de venta de vehículos japoneses.

Mientras que se necesita 10 veces el gasto promedio anual de un hogar en Colombia para adquirir una Tahoe, en Estados Unidos se necesita menos de 2 veces y en Japón aproximadamente 3. Es decir que un hogar promedio puede comprar este vehículo en Colombia (sin blindarlo) si ahorra el equivalente a 10 veces su gasto total anual. Se repite el ejercicio contrastando una Toyota Prado referenciada en la muestra del AMP 2019, pero ahora contra el PIB per cápita en dólares PPA-PPP, concluyendo que en Colombia el individuo promedio tendría que destinar casi 9 veces su ingreso anual para aspirar a pagar una camioneta de dicho modelo, mientras que en EE.UU y Japón, el valor no alcanza a llegar a 2.

A continuación, la Figura 3 incorpora un comparativo macroeconómico entre los mismos tres países, incluyendo el gasto promedio de hogares, el PIB per cápita, y los precios locales de algunos vehículos presentes en el AMP de 2019. 


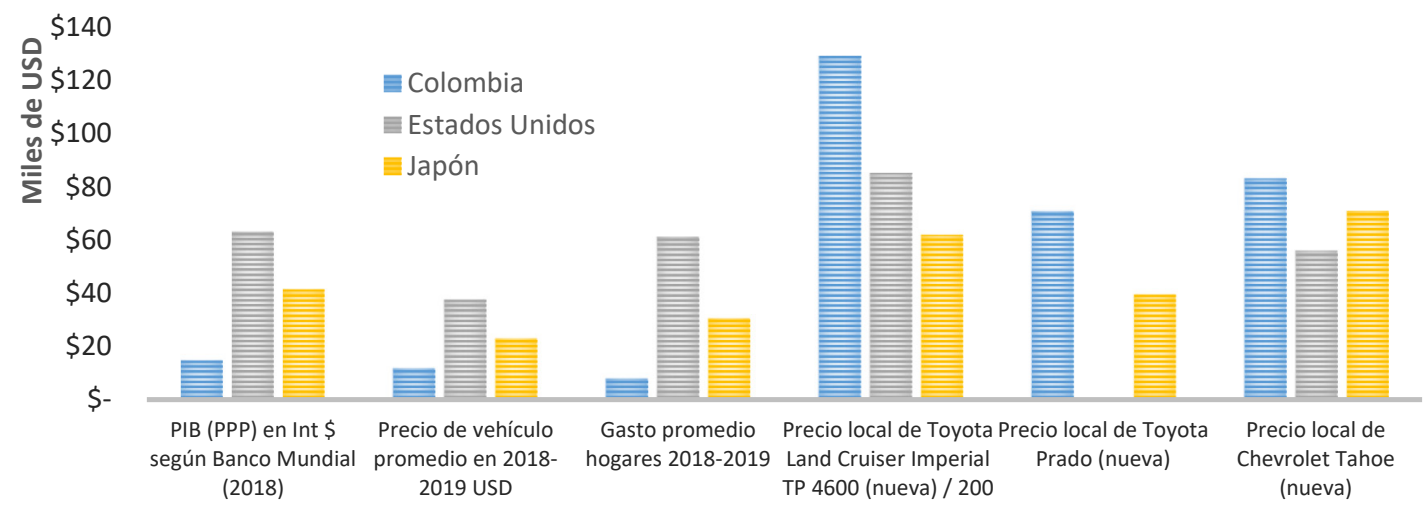

Nota: A la izquierda se percibe la brecha que tiene Colombia frente a EE.UU y Japón en PIB per cápita, gasto promedio de los hogares y en el costo de un vehículo promedio. A la derecha, se muestra el precio en USD de tres vehículos seleccionados. Mientras que las variables macro reflejan una falta de capacidad económica de Colombia, el costado derecho implica que los costos de los vehículos son comparativamente altos para la capacidad de la economía colombiana.

Fuente: Elaborada por el autor a partir de datos de DANE, Banco Mundial, Kelly Blue Book, Kakaku Japan, Tucarro.com, y varios portales de venta de vehículos japoneses. La Toyota Prado no se encuentra en EE.UU en el mismo modelo comparado.

La Figura 3 refleja la necesidad de preguntar no solo por el precio nominal del activo, sino por la capacidad promedio que tiene un hogar en el país de origen de la empresa y en el país destino. La gráfica sugiere que la economía colombiana tiene que hacer un esfuerzo ampliamente superior al de una economía industrializada, para financiar vehículos similares. A esta situación se suma el riesgo que implica la depreciación del peso colombiano, que materializa la pérdida del poder adquisitivo de los hogares y del Estado. En este caso vale la pena preguntar si hay una demanda perfectamente inelástica, pues el Estado no deja de realizar estas compras o alquileres, anclado en culturas del malgasto.

¿Es adecuado que el Estado colombiano financie vehículos de alta gama para el Estado, cuando no tiene la misma capacidad de compra de los países productores de dichos vehículos? Ante esta pregunta surge usualmente el argumento de garantizar la seguridad de la persona protegida como objetivo superior. Sin embargo, se podría desvirtuar si la misma misión se puede cumplir con vehículos más económicos, blindados con el mismo requerimiento del National Institute of Justice, i.e. el estándar internacional que aplica el Estado colombiano.

\subsubsection{Requerimientos de Blindaje y el Obstáculo de las Narrativas Técnicas}

Desde una de las listas de vehículos de referencia del proyecto, se toman 174 camionetas registradas en el AMP del 2019 para vehículos blindados, efectivamente ofrecidos, según CCE, con detalles de los oferentes y los modelos exactos. En contraste, encontrar el modelo exacto del vehículo en los documentos de SECOP para cada contrato, supone dificultades serias, motivo por el cual los AMP generan una opción de control al contrastar precios. Sin embargo, los diferenciales entre el AMP y los contratos reales también ofrecen otra capa de análisis que trasciende este propósito (cf. Behar- 
Villegas \& Amado, 2021). En el grupo de los 174 vehículos, solo aparecen 3 vehículos con un precio, antes del blindaje, inferior a los 30.000 USD (e.g. una Renault Duster Dynamique MT 2000CC 4X4 por 21.430 USD), efectivamente ofrecido como vehículo a blindar para el Estado.

En el AMP aparece el blindaje ofrecido, según el estándar NIJ, especificado en el documento técnico 0108.01 del National Institute of Justice. El blindaje oscila entre las categorías II y IV, especificando si se usa material liviano o pesado. El nivel IIIA, por ejemplo, debe resistir entre 500-1000 joules, equivalente a un proyectil calibre $9 \mathrm{~mm}$, mientras que el III debe resistir disparos de fusil calibre 7.62, i.e. 3400 joules. Un criterio esencial para el blindaje es la capacidad de carga del vehículo, a parte de otros criterios como la calidad de los frenos, el respaldo del fabricante, la relación peso/potencia, la vida útil esperada, etc. La presencia de vehículos menos costosos ofrecidos, blindables al mismo nivel de vehículos de alta gama, indica que es posible encontrarlos en el mercado. Contra este argumento se puede anteponer la vida útil de un vehículo pequeño, contra una camioneta de alta capacidad de carga como una Toyota Land Cruiser. Sin embargo, en la revisión de contratos recientes, se percibe una tendencia a arrendar por tres años y no comprar los vehículos. De ahí que el argumento de la vida útil sea prácticamente irrelevante cuando hay alquiler y no compra.

El proyecto de investigación se concentró en los vehículos de exigencia IIIA y III con materiales livianos y pesados, dejando de lado la más alta categoría, pues ésta implica riesgos extremos de ataques con explosivos para figuras como el Presidente de la República, los altos mandos militares, civiles amenazados, etc. Aquí surge una de las problemáticas anclada en la subjetividad de funcionarios que elaboran los estudios previos. Por un lado, están atados a contrataciones pasadas, con criterios que difícilmente modificarían. Por el otro, el nivel de riesgo se evalúa en la misma entidad (Unidad Nacional de Protección-UNP), que usualmente contrata o compra los vehículos, para ponerlos a disposición de las entidades según su necesidad. El nivel de riesgo se puede elevar desde la subjetividad y las relaciones políticas en las entidades, actuando bajo la narrativa de seguridad, que a su vez distorsiona la realidad técnica y de seguridad de individuos protegidos por el Estado. Si la UNP es la que revisa el riesgo y a la vez escribe los estudios previos, puede haber un efecto endogámico que distorsiona la calidad del gasto.

Así, se realizó un análisis de efectividad de costos como insumo para estudiar la eficiencia del gasto, que permite visualizar la brecha que habría entre un gasto óptimo (e.g. precios de mercado de vehículos de categoría inferior, que respetan el requerimiento técnico NIJ) y los precios del modelo exacto documentado por el AMP. Se compara entonces $P_{\text {proc }, i}$ como precio de compra (procurement) y $P_{m, c a t-1, i-z}$ como precio de categoría inferior. Siguiendo a Cellini \& Kee (2010), se calcula la razón de efectividad del costo (Cost Effectiveness Ratio - CER), utilizando la variable $s$ como unidad de efectividad, que en este caso corresponde a la vida salvada de la persona protegida, así:

$$
C E R=\frac{s}{\left(P_{p r o c, i}-\mathrm{P}_{m, i-z}\right)}, \text { si } P_{p r o c, i}-P_{m, i-z} \geq 0
$$

en donde CER, o índice de efectividad representa las vidas salvadas por diferencial de costos, fungiendo como parámetro de eficiencia. Si el delta entre los precios es alto, la vida se salva, pero a un costo más alto del que sería necesario dados los precios de mercado. Si el delta tiende a $\infty$, el CER tiende a 0 . Sí el delta tiende a 0 , se maximiza la efectividad al tender a $\infty$. Esto implica que la agencia compra o alquila el vehículo óptimo, bajo la premisa de salvar la vida según el criterio de blindaje. Si se agrega para una muestra de $n$ vehículos, se tiene el índice agregado: 


$$
C E A_{n}=\frac{s \cdot n}{\sum_{i=1}^{n}\left(P_{\text {proc }, i}-P_{m, i-z}\right)} \text {, si } P_{\text {proc }, i}-P_{m, i-z} \geq 0
$$

El resultado, tomando precios nominales en pesos colombianos, para la lista del AMP 2019, se presenta en la Figura 4. Cuantos más puntos aparecen cerca al eje X, menos efectividad hay en los costos, dado que la diferencia entre el precio de compra y el precio de mercado de una categoría inferior, técnicamente viable al respetar el requerimiento de blindaje, es considerable. En el caso de observar convergencia entre los precios, el indicador estará por encima de 1.

FIGURA 4 ÍNDICE DE EFECTIVIDAD EN REFERENCIAS DE VEHÍCULOS, N=174

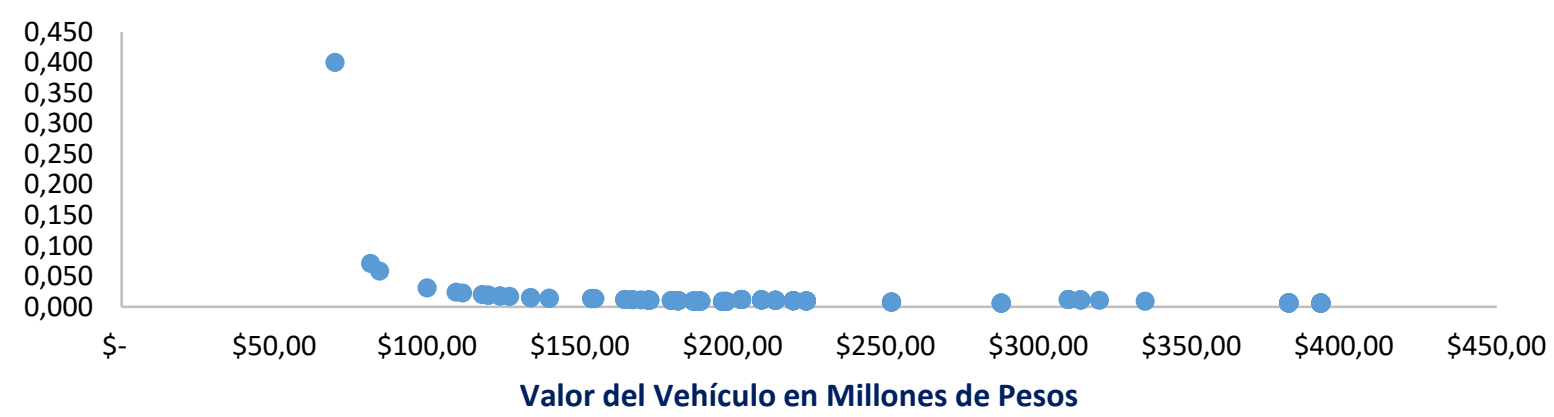

Nota: El índice muestra la brecha que hay entre los precios de compra y los precios técnicamente viables. Cuando más bajos los resultados, menor efectividad. Cuanto más altos, más convergencia entre precios, es decir que se compra la categoría de vehículo óptima, respetando el mínimo de requerimientos técnicos.

Fuente: Elaborada por el autor.

Para este grupo de referencia se calcula un valor promedio de ahorro de ca. 29.000 USD, si el Estado adquiere camionetas y camperos de categorías inferiores, técnicamente aceptables. Frente a este punto se evaluaron tres métodos. En el primero, se escoge una categoría inferior dado que se encuentra la oferta del vehículo inferior, con el mismo blindaje, de un proponente en el AMP. Es decir, si un proponente oferta una Renault Duster blindaje IIIA con materiales pesados, se asume que, al existir en el mercado, no es necesario comprar una Toyota Prado que requiere la misma especificación. Con el segundo método se controla por la capacidad de carga, mientras que para la categoría IV, no se toma el más económico, sino el siguiente vehículo en precio. En el método 3, se controla igual por la capacidad de carga y no se cambian los vehículos más costosos, partiendo de la premisa que el riesgo de ataque por explosivos es probable, y que el vehículo se usará en casos extremos como los mencionados arriba. De esta manera, se concentra el estudio solo en los cargos de servicio público más atomizados, mencionados arriba. El resultado de los tres métodos arroja un $\%$ de ahorro que oscila entre el 43 y el $49 \%$ del valor total de los 174 vehículos. Es decir que se podría cumplir la misma misión, comprando vehículos de categorías inferiores, generando ahorros cercanos a la mitad del agregado. Si se tiene en cuenta que esta referencia de valores no es de un contrato real, sino del acuerdo marco, existe aún más espacio para ahorrar, dado que los precios del AMP son el estándar que sugiere CCE. 


\subsubsection{Culturas del Gasto y narrativas latentes}

¿Por qué se compran vehículos de alta gama que generan malgasto? Una respuesta se puede dar desde las culturas del malgasto, atadas al incrementalismo, a expectativas estéticas, al sesgo por el presente, a la narrativa de la seguridad, y a otros factores que pueden construir una inercia del malgasto de la que no necesariamente hay conciencia.

Por un lado, el ejercicio presupuestal está anclado en el pasado, en las expectativas y en la narrativa de seguridad, que maximiza el tipo de vehículo mas no la eficiencia en el gasto al incorporar medidas de efectividad de costos. Por otro lado, habría expectativas estéticas sobre los artefactos que están asociados a altos cargos, creando una estética de poder que busca igualar a aquella que se ve en el sector privado, en los productos de la industria del entretenimiento (e.g. caravanas blindadas en películas, etc.), y que a su vez ha sido influenciada por la narcoestética (Corcione, 2018).

La narcoestética implica el uso de símbolos de estatus, riqueza, poder y prestigio durante el auge de los carteles de la droga en Colombia, desde mediados de los años 80. A su vez, el uso de camionetas blindadas se puede trazar a los años 80 y 90 con la aparición de nuevos modelos de la Toyota Land Cruiser (e.g. FJ62LG) inicialmente de silueta más cuadrada, y luego conocidas como Burbujas desde 1993 (e.g. Autana). Con estos vehículos se forjó una narrativa de poder, paradójicamente en la tensión del poder del Estado y aquel que lo desafiaba desde los círculos del narcotráfico. El vehículo funciona entonces como un artefacto de protección, exclusión, inclusive como un escondite detrás de los vidrios oscuros, que forja costumbres que construyen expectativas de gasto. Resulta políticamente difícil imaginar el uso de vehículos más pequeños en altos cargos de poder, al crear una distorsión entre la estética inercial de la expectativa y la necesidad latente pero individualmente inconveniente de mejorar el gasto público.

Por otra parte, resulta importante mencionar el tema de los alquileres (leasing). En los contratos revisados en el proyecto (Behar-Villegas \& Amado, 2021), en especial en los de la UNP, se encuentra que el Estado paga entre 2.500 y 4.500 USD mensuales por vehículo, durante tres años, es decir entre 9 y 17 veces el salario mínimo legal en Colombia. El alquiler se argumenta desde el precio del mantenimiento, pero, tal y como se explicó para un contrato reciente de camionetas blindadas para la Judicatura, "los vehículos se están comprando ahora para ahorrar costos, ya que el alquiler de los mismos tiene un valor aproximado a los \$15 millones [ca. 4500 USD] y es más económico adquirir las camionetas por tener una proyección de uso de 10 años" (Revista Semana, 2020b). Esto indica que existe un grado de conciencia sobre la diferencia de estos dos escenarios. Alquilar por tres años supone que los funcionarios pueden estrenar vehículos frecuentemente. En contraste, en promedio se toman 16 años en renovar un vehículo en Colombia (La República, 2018).

La paradoja que se percibe aquí aparece en la idea de narrativas latentes con artefactos explícitos. Los vehículos cuentan la historia de la necesidad de protección, evidente ante la violencia que ha vivido Colombia. Son artefactos de storytelling paradójicos, porque la verdad del gasto, detrás del telón, no se conoce al ser técnica y jurídicamente difusa. La narrativa de la seguridad entonces sirve para sustituir preguntas posibles desde la eficiencia técnica, creando culturas incrementalistas que se materializan en la formulación de estudios previos que no incorporan una comprensión holística del problema del malgasto potencial. La narrativa es entonces latente, porque forja unas culturas o comportamientos aprendidos, de los cuales no hay conciencia. Si a esto se suma la rotación del personal que traen los ciclos políticos, difícilmente se pueden observar cambios en las tradiciones presupuestales, que absorben inclusive efectos de depreciación de la moneda local.

ReVISTA de AdMinistração PÚblica ｜ Rio de Janeiro 55(3): 662-678, maio - jun. 2021 
Al contar la historia del status y recordar estructuras de poder desde los altos cargos, los vehículos distancian al protegido del villano (la inseguridad), ya expuesto permanentemente en los medios a través de la violencia. Sin embargo, en dicho storytelling, no parece haber lugar para la contranarrativa que podría cambiar los artefactos por unos menores, al amenazar el estatus quo que impone la narrativa de la seguridad y sus narrativas técnicas. Paradójicamente, se anuncia con frecuencia la necesidad de reducir el gasto, pero dicha historia no se absorbe técnicamente en los escenarios (settings). Esos lugares están imbuidos de culturas del malgasto.

\section{CONCLUSIÓN}

Las culturas del malgasto público engloban comportamientos aprendidos en la gestión administrativa, que violan los principios de eficiencia, efectividad y economía. Documentar estas culturas contribuye a exponer el rol y la consecuencia de las narrativas que operan en el sector público, alimentando comportamientos y tradiciones que son política y metodológicamente difíciles de cuestionar. En este trabajo se ejemplificaron dichas culturas en las prácticas de compra y alquiler de vehículos de alta gama para el Estado. La inercia en las compras públicas puede estar protegida por narrativas que difícilmente se pueden identificar en el día a día del sector público. El caso presentado apunta a una desconexión entre principios de la administración pública (e.g. eficiencia) con la realidad, que está permeada por culturas del malgasto. Se sugiere que las narrativas alimentan las culturas del malgasto público, volviendo herméticas e inerciales las prácticas aprendidas a través de la falta de transparencia en la información y los detalles técnicos, que contribuyen a la ineficiencia estatal.

Este estudio representa una contribución inicial a un tema que requiere más esfuerzos interdisciplinarios para caracterizar y documentar las culturas del malgasto. Se registra apenas una muestra referencial con la limitación clara de no poder incorporar información de todas las compras y alquileres de carros blindados. Sin embargo, el principio de reconocer posibles escenarios de culturas del malgasto se puede extrapolar para explorar caso por caso aquello que sucede en las compras públicas, y así generar marcos generales de replicabilidad. En ello tienen una responsabilidad y una ventaja fundamental los organismos de control (e.g. Contralorías), que paradójicamente, al politizarse, pierden capacidades y experticia para detectar estos detalles. Se hace necesario que los organismos de control usen marcos de análisis técnicos con funcionarios altamente calificados en temas de ingeniería mecánica, análisis de costo-beneficio, i.a, según el sector, pero que a la vez tengan una comprensión holística de los problemas, para que no se tomen decisiones desacertadas que generen más comportamientos incrementalistas y desincentiven la presencia de compradores expertos.

Otra dificultad en la revisión de estos procesos se da en el rol que pueden jugar los actores de mercado, i.e. las marcas y los consorcios, al preferir vender vehículos de precios más altos. Sin embargo, el proyecto marco en el cual nace este trabajo identifica que las empresas blindadoras no necesariamente tienen un incentivo de que se vendan o arrienden carros más grandes o más lujosos, pues pueden ofrecer sus mismos servicios para blindar carros más pequeños. Este punto, sin embargo, requiere futuros esfuerzos de investigación.

Por otro lado, no se puede descartar que la preferencia de los carros de alta gama esté relacionada con la dificultad del terreno en Colombia, pues algunas camionetas blindadas opera en zonas rurales, 
en donde se hacen necesarios carros con blindajes superiores s NIJ IV, debido a las amenazas contra agentes del Estado y líderes sociales.

La aproximación desde los estudios de caso se encuentra con la limitación de no poder integrar todas las narrativas que pueden estar influenciando las culturas del malgasto, dando apenas un ángulo de observación a un tema que requiere trabajos futuros. Es importante identificar demás narrativas (e.g. la seguridad, el progreso, el control fiscal, etc.) que pueden frenar una mirada interdisciplinaria a la asignación del gasto en otros sectores. Por ejemplo, al decir que se requieren carros blindados para proteger la integridad de un servidor público, la cultura del malgasto, anclada en el incrementalismo presupuestal, difícilmente permite que se increpe si un tipo de vehículo es realmente necesario para cumplir la misma misión. 


\section{REFERENCIAS}

Banco Interamericano de Desarrollo. (2018, septiembre 24). Gasto público en América Latina registra ineficiencias del $4.4 \%$ del PIB: estudio BID. Recuperado de https://www.iadb.org/es/ noticias/gasto-publico-en-america-latina-registraineficiencias-de-44-del-pib-estudio-bid

Bandiera, O., Prat, A., \& Valletti, T. (2009). Active and Passive Waste in Government Spending: evidence from a Policy Experiment. American Economic Review, 99(4), 1278-1308.

Behar-Villegas, E. (2018, septiembre 28). Cortoplacismo egocéntrico o visión de largo plazo: no más marcas de gobierno. Revista Dinero. Recuperado de https://www.semana.com/opinion/ columnistas/articulo/no-mas-marcas-de-gobiernopor-erick-behar/262619/

Behar-Villegas, E., \& Amado, P. (2021). Devil in the details and public value: a cost-effectiveness analysis of wasteful government spending using armored cars in Colombia (Working Paper). Berlin, Germany: Berlin International University of Applied Sciences.

Cantera, S. (2018, septiembre 02). Los autos blindados, tocados por López Obrador. El Economista America.com. Recuperado de https:// www.eleconomistaamerica.com/economia-eAmmexico/noticias/9361716/09/18/Los-cochesblindados-tocados-por-Lopez-Obrador.html

Cellini, S. R., \& Kee, J. E. (2010). Cost-Effectiveness and Cost-Benefit Analysis. In J. S. Wholey, H. P. Hatry, \& K. E. Newcomer (Eds.), Handbook of Practical Program Evaluation (3a ed., pp. 493-530). San Francisco, CA: Jossey-Bass.

Citizens Against Government Waste. (2015). Changing the culture of Wasteful Spending. Recuperado de https://www.cagw.org/thewastewatcher/changingculture-wasteful-spending

Colombia Compra Eficiente. (2019). Listado de Vehículos de Referencia para el Acuerdo Marco de Precios LP-AMP-137-2017 para la adquisición de Vehículos Blindados (Versión 15). Recuperado de https://www.colombiacompra.gov.co/tienda-virtualdel-estado-colombiano/transporte/vehiculosblindados-ii

Corcione, J. S. (2018). Narcoestética: El gusto narco en Colombia en la década de los años ochenta y noventa
(Tesis de Maestría). Universidad De Bogotá Jorge Tadeo Lozano, Bogotá, Colômbia.

Cormann, S. (2013). The Difference between Story and Narrative. Tempe, Arizona: Center for Strategic Communication, Arizona State University. Recuperado de https://csc.asu.edu/2013/03/21/thedifference-between-story-and-narrative/

Dal Bó, E., \& Rossi, M.A. (2007). Corruption and inefficiency: Theory and evidence from electric utilities. Journal of Public Economics, 91(5-6), 939-962.

Doimeadiós Reyes, Y., \& Rodríguez Llorian, E. (2015). Un análisis comparado de eficiencia y eficacia en el sector público en Cuba. Economía y Desarrollo, 155(2), 44-59.

El Mostrador. (2008, marzo 5). Gobierno insta a evitar derroche al celebrar día de Eficiencia Energética. Recuperado de https://www.elmostrador.cl/noticias/ pais/2008/03/05/gobierno-insta-a-evitar-derrocheal-celebrar-dia-de-eficiencia-energetica/

Fiscalía General de la Nación. (2020, marzo 19). Fiscalía y Contraloría anuncian acciones contundentes para contener efectos del Coronavirus en el país. Recuperado de https://www.fiscalia.gov.co/ colombia/noticias/fiscalia-y-contraloria-anuncianacciones-contundentes-para-contener-efectos-delcoronavirus-en-el-pais/

French, K., Shanahan, E.A., Raile, E.D., \& McEvoy, J. (2017). Narrative Frames and Settings in Policy Narratives. In Proceedings of the $3^{\circ}$ International Conference on Public Policy, Singapore. Recuperado de https://www.ippapublicpolicy.org/file/ paper/59441c8498920.pdf

Gallego, J., Rivero, G., \& Martínez, J. (2020). Preventing rather than punishing: An early warning model of malfeasance in public procurement. International Journal of Forecasting, 37(1), 360377. Recuperado de https://doi.org/10.1016/j. ijforecast.2020.06.006

Geertz, C. (1973). The Interpretation of Cultures. New York, NY: Basic Books.

González Gaitán, J.A. (2020, julio 17). Contratos cuestionados de Fiscalía y Contraloría en medio del coronavirus. Caracol Radio. Recuperado de https://caracol.com.co/radio/2020/07/17/ judicial/1595009134_983996.html 
Hannerz, U. (2010). Anthropology's World. Life in a Twenty-First-Century Discipline. London, UK: Pluto Press.

Hauptmeier, S., Heinpertz, M., \& Schuknecht, L. (2006). Expenditure Reform in Industrialised Countries. A case study approach (Working Paper Series, 634). Frankfurt, Germany: European Central Bank.

Hiebert, P. (1997). Cultural Anthropology (2a ed.). Grand Rapids, MI: Baker Book House.

Holdsworth, M. (2019). Wasteful Spending in Health Care: A US and UK international comparison. French Journal of British Studies, 24(3), 1-14. Recuperado de https://doi.org/10.4000/rfcb.4381

Izquierdo, A., \& Pessino. (2018). La (in)eficiencia del gasto público. In A. Izquierdo, C. Pessino, C., \& G. Vuletin (Eds.), Mejor gasto para mejores vidas. Cómo América Latina y el Caribe puede hacer más con menos. Washington, DC: Banco Interamericano de Desarrollo.

Jenofonte. (1997/s.d.). Memorabilia (Erinnerungen an Sokrates). Leipzig, Germany: Reclam Verlag.

Jones, M. D., \& McBeth, M. K (2010). Narrative Policy Framework: clear enough to be wrong? Policy Studies Journal, 38(2), 329-353.

Jones, M. D., McBeth, M. K., \& Shanahan, E. A. (2014) Introducing the Narrative Policy Framework. In M. D. Jones, E.A. Shanahan, \& M. K. McBeth (Eds.), The Science of Stories. London, UK: Palgrave Macmillan.

La República. (2018, abril 27). Un colombiano cambia su carro particular en promedio cada 16 años. Recuperado de https://www.larepublica. co/empresas/mas-de-53-del-parque-automotorsupera-los-10-anos-2719394

Licht, A. N., Goldschmidt, C., \& Schwartz, S. H. (2005). Culture, Law, and Corporate Governance. International Review of Law and Economics, 25(2), 229-255.

Liebman, J. B., \& Mahoney, N. (2018). Do Expiring Budgets Lead to Wasteful Year-End Spending? Evidence from Federal Procurement (NBER Working Paper No. 19481). Cambridge, MA: National Bureau of Economic Research.

Lowndes, V. (2016). Storytelling and narrative in policymaking. In G. Stoker, \& M. Evans (Eds.),
Evidence Based Policymaking in the Social Sciences: Methods that Matter (Chap. 6.). Bristol, UK: Policy Press.

Mastroianni, G. R. (2006). Occupations, Cultures, and Leadership in the Army and Air Force. Parameters: US Army War College Quarterly, 35(4), 76-90.

McKevitt, D. (2015). Debate: value for money in search of a definition. Public Money and Management, 35(2), 99-100.

Mishler, E. (1995). Models of narrative analysis. Journal of Narrative and Life History, 5(92), 87-123.

Montoia, V. (2019, junho 17). Como são os 29 carros de luxo que Bolsonaro encomendou para sua família e do vice Mourão; 17 deles blindados. Auto Esporte. Recuperado de https://autoesporte.globo.com/ carros/noticia/2019/06/como-sao-os-29-carros-deluxo-que-bolsonaro-encomendou-para-sua-familiae-do-vice-mourao-17-deles-blindados.ghtml

Moosmüller, A. (2000). Die Schwierigkeit mit dem Kulturbegriff in der Interkulturellen Kommunikation. In R. Alsheimer, A. Moosmüller, \& K. Roth (Eds.), Lokale Kulturen in einer globalisierten Welt (Münchener Beiträge zur Interkulturellen Kommunikation, Vol. 9, pp. 15-31). Münster, Germany: Waxmann Verlag.

Ouchi, W. (1980). Markets, Bureaucracies, and Clans. Administrative Science Quarterly, 25(1), 129-141.

Payne, J. L. (1991). The Culture of Spending: why Congress Lives Beyond our Means. San Francisco, CA: Institute for Contemporary Studies.

Polletta, F. (2008). Storytelling in Politics. Contexts, 7(4), 26-31.

PwC. (2012). Good budgetary processes: comparators. Case studies from the public and private sector (PwC research Report/National Audit Office). Recuperado de https://www.nao.org.uk/wp-content/ uploads/2012/10/NAO_Good_budgeting_Research. pdf

Revista Semana. (2020a, febrero 6). Duque emite directiva para evitar derroche en contratos del Gobierno. Recuperado de: https://www.semana.com/ semana-tv/semana-noticias/articulo/directiva-paraevitar-derroche-en-contratos/675625 
Revista Semana. (2020b, marzo 28). Judicatura compró 51 camionetas blindadas en época de covid-19. Recuperado de https://www.semana.com/ semana-tv/semana-noticias/articulo/coronavirusjudicatura-compro-51-camionetas-blindadas-enepoca-de-covid-19/666670

Rocha Menocal, A., \& Taxell, N. (2015). Why corruption matters: understanding causes, effects and how to address them. Evidence Paper on Corruption. London, UK: UK Department for International Development. Recuperado de https://assets. publishing.service.gov.uk/government/uploads/ system/uploads/attachment_data/file/406346/ corruption-evidence-paper-why-corruptionmatters.pdf

Rodríguez, C. (2019). La 'administración paralela' de la Consejería de Cultura: 24 millones de presupuesto y casi todo para nóminas. El Mundo. Recuperado de https://www.elmundo. es/andalucia/2019/06/17/5d07b7d4fc6c83ea77 8b465d.html

Rose-Ackerman, S. (1997). Corruption, inefficiency and economic growth. Nordic Journal of Political Economy, 24(1), 3-20.
Rueda López, N. (2011). La eficiencia y su importancia en el sector público. eXtoikos, 1, 48-47.

Schank, R.C., \& Abelson, R.P. (1977). Scripts, Plans, Goals, and Understanding: an Inquiry into Human Knowledge. New York, NY: Lawrence Erlbaum Associates.

Schein, E. H. (1990). Organizational culture. American Psychologist, 45(2), 109-119. Recuperado de https://doi.org/10.1037/0003-066X.45.2.109

Shenggen, F. (2007). Case Study \#9-2 of the Program: 'Food Policy for Developing Countries: The Role of Government in the Global Food System. In P. Pinstrup-Andersen, \& F. Cheng (Eds.), Food Policy for Developing Countries: Case Studies (pp. 1-16). Ithaca, New York: Cornell University Press. Recuperado de https://ecommons.cornell.edu/ handle/1813/55724

Stake, R. (1995). The Art of Case Study Research. London, UK: SAGE.

Stone, D. (1988). Policy Paradox. The art of Political Decision Making (3a ed.). New York, NY: Norton \& Company.

\section{Erick Behar-Villegas}

https://orcid.org/0000-0001-8714-4602

Decano y profesor de Economía en la Faculty of Business Administration of Berlin International University of Applied Sciences. E-mail: behar@berlin-international.de; erick.behar@hotmail.com 